\title{
ANÁLISIS DEL USO DE UN GLOSARIO PARA LA LECTURA DE TEXTOS CIENTÍFICOS EN UNA LENGUA EXTRANJERA
}

\author{
Marcos Peñate \\ Alejandro Rodríguez
}

\begin{abstract}
RESUMEN
En los casos en que el alumnado universitario tiene que estudiar una asignatura de su carrera, usando solo libros escritos en una lengua extranjera, el disponer de un nivel aceptable de lectura se convierte en condición fundamental. No obstante, en el presente artículo se plantea que no es suficiente dicho nivel y que el alumnado deberá disponer también de un glosario de términos específicos.

Empezamos haciendo un breve resumen de la línea de investigación que se ha interesado por conocer cuáles son los factores que tienen una mayor incidencia a la hora de leer en una segunda extranjera. A continuación presentamos un estudio, llevado a cabo con el alumnado de Oceanografía Física de la Universidad de Las Palmas de Gran Canaria, con el que pretendemos analizar las ventajas del uso de un glosario de términos específicos.

Palabras clave: lectura, lengua extranjera, fines académicos, investigación, glosario.
\end{abstract}

\begin{abstract}
One of the basic conditions required for university students to study a subject, using only books written in a foreign language, is that they have an acceptable level of reading. Nevertheless, we have tried to prove that having such a level is not enough and that students need an extra tool: a glossary of the specific terms used in the study area.

This paper begins by giving a brief outline of the research carried out into how foreign language students reach an acceptable level of Reading. Then we present a case study made with students of Physical Oceanography at the University of Las Palmas de Gran Canaria (Spain), in which we try to prove the advantages of using a glossary of specific terms.

Key words: reading, foreign language, academic purposes, research, glossary.
\end{abstract}

\footnotetext{
Marcos Peñate. Profesor Titular. Didáctica de la Lengua Extranjera. Universidad de Las Palmas, Gran Canaria. Coreo electrónico: mpenate@dde.ulpgc.es

Alejandro Rodríguez. Profesor Asociado. Lengua Inglesa. Universidad de Las Palmas, Gran Canaria.

Correo electrónico: arodsua@hotmail.com
}

Recepción: 10-2-2009

Aceptación: 20-3-2009 


\section{Introducción}

Son numerosas las investigaciones que han analizado cómo los estudiantes de una lengua extranjera (LE) consiguen alcanzar un nivel de lectura adecuado. En los estudios realizados, como se puede ver en el artículo recopilatorio de Rodríguez y Peñate (2008), nos encontramos con dos enfoques perfectamente delimitados. Unos (Berman 1984; Cooper 1984, Segalowitz 1991) defendían que la habilidad lectora en una lengua extranjera está estrechamente condicionada y determinada por el nivel lingüístico que el alumno tenga en dicha lengua, mientras que otros (Clarke 1980; Block 1986, 1992; Upton 1997) mantenían que las estrategias lectoras que el alumno ha adquirido en su lengua materna (LM) son transferidas a la segunda lengua.

Investigadores pertenecientes a la primera corriente (Rayner y Pollastek 1989; Stanovich 1986; Daneman 1996, entre otros) vienen a defender un procesamiento de la lectura ascendente, puesto que consideran que la lectura es un proceso que comienza con el reconocimiento de palabras, pasando por las frases y así hasta llegar a procesos lectores más avanzados. Según este punto de vista, todo el desarrollo se verá impulsado por la competencia lingüística que el alumno vaya adquiriendo.

La segunda corriente, la que entiende a la lectura como competencia estratégica, ha analizado diferentes aspectos. Así, Cummins (1980) observa que la competencia cognitiva es común en la LM y la LE, del mismo modo que Clarke (1980) concluye que existe cierta transferencia entre los buenos lectores de LM y LE, aunque Clarke termina sugiriendo que son las conductas los verdaderos factores que determinan la lectura eficaz e ineficaz. Otro autores más recientes (como Upton 1997) testifican que el lector de LE siempre está condicionado por su LM y que la traducción desempeña un papel trascendental en la lectura.

No obstante, estas dos corrientes iniciales terminan por confluir y ello da lugar a un tercer modelo llamado el modelo interactivo. Trabajos posteriores (Cornaire 1999:24) han venido a ratificar que el enfoque interactivo es mucho más flexible que los modelos basados solo en las teorías que defienden la lectura como competencia linguiística, por un lado, o los que amparan la competencia estratégica, por otro.

Un trabajo que se había planteado de forma directa la cuestión de si la lectura en una LE depende de la habilidad lectora o del nivel de lengua fue el de Carrel (1991). Este estudio de tipo experimental constata que ambos factores inciden de manera directa en la destreza lectora, siendo esta conclusión semejante a la de otro trabajo posterior (Bernhardt y Kamil 1995). Estos últimos, tras estudiar a 186 nativos ingleses que leyeron textos en inglés y en español, probaron que los resultados indicaban que ni la hipótesis del umbral lingüístico ni la de la interdependencia lingüística reflejaban totalmente el proceso lector de las segundas lenguas. La primera viene a establecer que el conocimiento gramatical y terminológico es crucial e indispensable para poder llegar a procesos lectores más avanzados, mientras que la segunda hipótesis plantea que las operaciones lingüísticas como la lectura y la escritura son transferibles de una lengua a otra y que están entrelazadas. Concluyen los autores que ambas hipótesis llegan a ser ciertas y erróneas dependiendo de qué caso se trate. A conclusiones similares llegó el estudio de Huntley y Peñate (2003) en el que se constata que el alumnado solo puede hacer uso de su competencia estratégica cuando se enfrenta a textos de niveles no muy superiores al que ellos disponen, y cuando el nivel del texto es mayor, el uso de estrategias deja de ser relevante. 
En el caso que nos ocupa, la lectura atenta de textos especializados, consideramos que los trabajos de investigación que realmente nos aportarán información relevante son los que se han centrado en estudiantes universitarios con niveles intermedios de LE y que incluyen terminología especializada puesto que, tal como nos recuerdan Crow y Quigley (1985: 510), la falta de vocabulario pasivo constituye un serio problema para el alumnado lector de textos universitarios en LE. Por tanto dedicaremos el resto de la presente introducción al análisis de algunos de estos estudios.

En el trabajo de Williams y Dallas (1984), se llevó a cabo un estudio piloto de lectura a estudiantes nigerianos y chinos en 1979 y se intentó arrojar luz sobre ciertos problemas de lectura existentes sobre todo en aquellos alumnos que pasan de estudiar inglés como asignatura a estudiarlo como medio de enseñanza. Es, entonces, cuando tendrán que afrontar las típicas dificultades conceptuales de la Ciencia, por un lado, y el estudio de la materia a través del medio de la lengua inglesa. El estudio se centró en un aspecto concreto como fue el vocabulario. Este se analizó desde distintas perspectivas en antiguos libros de texto de la Editorial Longman (una serie de nueve libros llamados Living Hong Kong Social Studies). Se observó el vocabulario en algunas definiciones presentes en ediciones antiguas, analizándose si estas definiciones servían o no de apoyo a los lectores. Se comprobó que había muchas dificultades con éstas, lo que llevó a los investigadores a incluir otras técnicas en ediciones posteriores tales como traducciones intratextuales, ilustraciones, cambio de clases de palabras, crucigramas y preguntas de elección múltiple.

Barnett (1986) estudió en su trabajo el papel que desempeña el vocabulario en la comprensión lectora. Realizó una prueba experimental a 124 estudiantes que cursaban francés en la Universidad de Virginia, de la que extrajo algunas de las siguientes conclusiones. Recoge que el apropiado dominio del léxico y de la sintaxis llega a afectar a la comprensión lectora. Comprobó que a mayor cantidad de vocabulario asimilado, mayor era el recuerdo de la información, lo que le indicó que sendas subdestrezas (vocabulario y sintaxis) resultaban indispensables en su experimento, constatando, al unísono, la interacción existente entre éstas.

Establece Ward (1999) que se ha extendido la idea de que los estudiantes necesitan de un listado de por lo menos tres mil palabras de las que un elevado porcentaje pertenece al campo del vocabulario genérico para llegar a poseer una comprensión aceptable de los textos. En un trabajo con estudiantes de primero de la carrera de Ingeniería en la Universidad de Suranaree de Tailandia, confeccionó una taxonomía de vocablos partiendo de una selección de textos y manuales de las diferentes disciplinas de esta carrera y de los listados léxicos ya existentes. El autor descubre que el alumnado puede llegar a desenvolverse perfectamente con familias de solo 2000 términos eminentemente técnicos, que no hay necesidad de que tengan que afrontar, en primera instancia, textos de conocimientos 'generales' y que los textos específicos no sobrecargan a los alumnos que han de estudiar terminología técnica difícil. Esto le sugiere que la lectura en LE para fines académicos en Ingeniería podría acometerse mucho antes. Asegura también que el léxico que desconoce el alumnado, formado generalmente por muchos vocablos compuestos, es en gran parte técnico y sugiere que se debe evitar facilitar términos irrelevantes al explicar las relaciones de los términos semantizados con la totalidad de la oración, y centrarse más en los relevantes, además de buscar preferiblemente objetivos específicos y homogéneos.

$\mathrm{Hu}$ Hsueh-chao et al. (2000) centran su trabajo experimental en analizar el nivel porcentual indispensable para lograr una comprensión ideal de textos de ficción. Con una 
muestra de 66 estudiantes adultos (tailandeses, chinos, vanuatus, indoneses, japoneses, coreanos, vietnamitas y alemanes) demostró que, efectivamente, la densidad de palabras desconocidas en textos literarios (a diferencia de los divulgativos o académicos) puede afectar al grado de entendimiento. Tanto es así que se averiguó que a medida que aumenta el número de vocablos desconocidos, peor es el entendimiento general. Constató que en términos globales, se tiene que conocer el $98 \%$ de palabras a fin de alcanzar una comprensión adecuada, sin interrupciones y sin tener que recurrir a glosarios.

He Ko (2005) investigó cómo diferentes tipos de ayudas en forma de glosarios afectan a la comprensión lectora de estudiantes universitarios coreanos. El autor estudió una muestra de 106 universitarios. A estos estudiantes se les pasó un material que presentaba tres condiciones distintas (129): sin ayuda de glosario, glosario en coreano (LM) y glosario en inglés (LE). Tras concluir la lectura, se pidió alumnado que realizara un cuestionario (cloze test con 25 ítems) y un test de elección múltiple (25 ítems). Los resultados indicaron que solamente el glosario en LE afectó de manera significativa a la comprensión lectora. No obstante, los protocolos de pensamiento en voz alta revelaron que ambos tipos de glosarios mejoraron y agilizaron la comprensión lectora.

\section{Objetivos}

Queremos iniciar este apartado especificando que partimos de la hipótesis de que el alumnado de esta asignatura de Oceanografía Física tiene una deficiencia en la capacidad de lectura comprensiva de textos escritos en lengua inglesa. Se postula pues que si los textos ingleses afectan al entendimiento de la materia provocando resultados deficientes, un glosario de términos específicos donde se explique brevemente el contexto puede hacer que los sujetos eleven su nivel de comprensión. Consecuentemente, si el bajo nivel de comprensión lectora en la materia de la Oceanografía Física se debe, entre otros aspectos, a que existe una barrera de comprensión de los textos ingleses que ha de leer el alumnado, nuestro objetivo será tratar de indicar qué factores determinan el nivel de comprensión de los textos en inglés en la materia científica de la Oceanografía Física. Es decir, trataremos de averiguar si bien se trata de la barrera lingüística propiamente dicha o del desconocimiento de la materia en sí.

\section{Sujetos}

Los sujetos de nuestra investigación fueron 43 alumnos que cursaban la asignatura de Oceanografía Física del primer curso de la Facultad de Ciencias del Mar de la Universidad de Las Palmas de Gran Canaria (España).

\section{Diseño de la investigación y materiales}

El diseño de la investigación se dividió en dos fases. En la primera se pasó un test intermedio de lectura a todo el alumnado. El nivel viene determinado por dos factores: el nivel lingüístico que se le pide al alumnado al entrar a la universidad (B1 del Marco Europeo de Referencia para las Lenguas equivalente al PET de Cambridge) y también por considerar que el alumnado debe ser capaz de entender textos de ese nivel para poder afrontar la lectura de textos especializados. 
Con los datos obtenidos en la prueba de inglés, se procedió a la formación de dos grupos de alumnos de forma aleatoria (el experimental y el control) garantizando que no hubiera diferencias estadísticamente significativas entre ellos. Para garantizarlo se ordenaron a los alumnos según las notas alcanzadas y asignando los impares al grupo control y los pares al grupo experimental. Ambos grupos realizaron una prueba de lectura específica bajo dos situaciones diferenciadas (con y sin glosario específico). Con los datos resultantes se hizo un análisis de tipo estadístico de las medias alcanzadas en cada grupo para comprobar si había diferencias estadísticamente significativas entre ambos. También se hizo un estudio de correlaciones entre los resultados de las dos pruebas de lectura: general y específica.

Los materiales que fueron utilizados vinieron determinados por el tipo de test de lectura realizado en cada caso.

\subsection{Test de lectura general}

Este test de lectura fue extraído de un examen de PET (Ward 2000: 135). Dentro de dicho examen se evalúa la destreza lectora a través de cuatro actividades. La primera mide la comprensión de anuncios públicos, señales o etiquetas. La segunda evalúa la comprensión global pero detallada de ocho pequeños textos. En la tercera actividad el alumnado deberá mostrar su habilidad para extraer información específica de cinco textos y, por último, la cuarta parte se centra en la capacidad de captar la opinión y la actitud del escritor.

\subsection{Test de lectura específica}

Para esta prueba se diseñaron dos tareas. En la primera el alumnado debía hacer un resumen en su lengua materna de cada uno de los siete párrafos del texto seleccionado, el cual versaba sobre los conceptos oceanográficos de masas de agua y circulación termohalina. Mientras que en la segunda, el alumnado debía ubicar diez términos dados. Los textos utilizados fueron tomados de la bibliografía básica de la asignatura con la ayuda del profesorado de la materia en cuestión. Para la selección de los términos que conformaron el glosario se utilizó como referencia los dos siguientes listados: el General Service List ${ }^{l}$ y el Academic Word List ${ }^{2}$. Es decir, en nuestro glosario solo se incluyeron palabras que no aparecen ni en el listado de carácter general ni tampoco en el listado que recoge los términos académicos comunes a todas las áreas y consecuentemente de mayor uso. Una muestra de ello, son los párrafos que se indican a continuación y que pertenecen a la primera actividad de lectura. En este caso el grupo experimental tiene como ayuda adicional el glosario de términos específicos, mientras que el grupo control recibió los mismos documentos pero sin esa ayuda adicional.

In most ocean regions the wind-driven circulation ${ }^{3}$ does not reach below the upper kilometre of the ocean.

Water renewal below that depth is achieved by currents which are driven by density differences produced by temperature (thermal) and salinity (haline) effects. The associated circulation is therefore referred to as the thermohaline circulation ${ }^{4}$. 


\footnotetext{
${ }^{3}$ Wind-driven circulation: Circulación superficial del océano cuya fuerza de impulsión es principalmente el viento.

${ }^{4}$ Thermohaline circulation: Circulación de la masa de agua oceánica cuya fuerza de impulsión se deriva de la diferencia de densidades.
}

\section{Resultados}

Empezaremos presentando los resultados obtenidos en la prueba de lectura de textos de tipo general, con la que se pretendía determinar el nivel de competencia lectora del alumnado. Posteriormente se detallarán los datos referidos a la prueba de lectura específica y los resultados de los diferentes análisis estadísticos.

\subsection{Resultados de la prueba inicial de lectura}

Como ya explicamos en el apartado dedicado a la metodología, la prueba inicial de inglés constaba de cuatro actividades de lectura. Todas tenían cinco ítems a excepción de la tercera que tenía diez, lo que nos da una suma total de 25 ítems para esta prueba inicial. Los resultados globales (Tabla 1) nos indican claramente que este alumnado tiene un nivel de lectura medio-bajo, pues solo uno (el 2,3\%) de ellos obtuvo la mayor puntuación (24 ítems acertados), siendo la peor nota aquella que alcanzó un estudiante con solo 10 ítems acertados. Los resultados más elevados obtenidos son los de 18 y 17 con 8 y 7 estudiantes (18,6\% y 16,3\%, respectivamente). Ahora bien, no se observó una clara tendencia bajista en el nivel de resultados pues se observa, al mismo tiempo, que existe un $11,6 \%$ con 20 aciertos y 22 aciertos y un $7 \%$ con 23 aciertos.

Tabla 1. Primer test de lectura

\begin{tabular}{|cc|r|r|r|r|}
\hline & & & \multicolumn{1}{|c|}{$\begin{array}{c}\text { Porcentaje } \\
\text { válido }\end{array}$} & $\begin{array}{c}\text { Porcentaje } \\
\text { acumu lado }\end{array}$ \\
\hline Válidos & 10,00 & 1 & 2,3 & 2,3 & 2,3 \\
& 15,00 & 5 & 11,6 & 11,6 & 14,0 \\
16,00 & 2 & 4,7 & 4,7 & 18,6 \\
17,00 & 7 & 16,3 & 16,3 & 34,9 \\
18,00 & 8 & 18,6 & 18,6 & 53,5 \\
19,00 & 2 & 4,7 & 4,7 & 58,1 \\
20,00 & 5 & 11,6 & 11,6 & 69,8 \\
21,00 & 4 & 9,3 & 9,3 & 79,1 \\
22,00 & 5 & 11,6 & 11,6 & 90,7 \\
23,00 & 3 & 7,0 & 7,0 & 97,7 \\
24,00 & 1 & 2,3 & 2,3 & 100,0 \\
Total & 43 & 100,0 & 100,0 & \\
\hline
\end{tabular}


Estos resultados nos permitieron, además de ver cuál era el nivel general de lectura de los sujetos de nuestra investigación, formar el grupo control y experimental siguiendo el criterio ya explicado en el apartado de metodología: ordenación del alumnado, según la puntuación obtenida en la prueba inicial y distribución aleatoria (impares, al grupo control y pares, al experimental). Una vez hecha la distribución y tras haber incorporado los datos al programa estadístico SPSS se pasó a realizar un análisis estadístico de comparación de medias, en concreto la prueba $\mathrm{T}$ de muestras independientes.

Antes de darnos los resultados de la prueba $\mathrm{T}$ de muestras independientes, el programa SPSS nos da las medias, desviaciones típicas y el error típico de la media tal como podemos apreciar en la siguiente tabla:

Tabla 2. Estadísticas de grupo

\begin{tabular}{|ll|r|r|r|r|}
\hline & GRUPO & N & Media & $\begin{array}{c}\text { Desviación } \\
\text { típ. }\end{array}$ & $\begin{array}{c}\text { Error típ. de } \\
\text { la media }\end{array}$ \\
\hline Primer test de lectura & experimental & 21 & 19,0476 & 2,63583 &, 57519 \\
& control & 22 & 18,4091 & 3,14202 &, 66988 \\
\hline
\end{tabular}

En la tabla precedente se puede comprobar que las medias, aunque no idénticas, son muy similares teniendo en cuenta que trabajábamos en una escala de 0 a 25 . No obstante, debíamos estar plenamente seguros de que no existían diferencias significativas entre las medias de los dos grupos que hemos formado de manera aleatoria. Para ello, acudimos a la prueba T de muestras independientes que nos dio los resultados que presentamos en la tabla 3.

Tabla 3. Prueba T de muestras independientes para la formación de los grupos

\begin{tabular}{lrr}
\hline & & Varianzas iguales \\
\hline Prueba de Levene para la igualdad de varianzas & F &, 164 \\
& Sig. &, 787 \\
\hline Prueba T para la igualdad de medias & T &, 720 \\
& Gl & 41 \\
& Sig. (bilateral) &, 675 \\
& Diferencias de medias &, 6385 \\
& Error típ de la diferencia & $-1,15660$ \\
& Intervalo de confianza inferior & 2,42906 \\
\hline
\end{tabular}

En esta tabla podemos apreciar que el programa ha hecho una prueba previa a la de comparación de medias. Al tratarse de grupos independientes, lo primero que hay que precisar es si las varianzas son iguales o no y para ello se utiliza la prueba de Levene. En este caso la prueba de Levene $F(0,164)$ tiene una probabilidad $(0,787)$ superior a 0,05 , lo que viene a indicarnos que no hay diferencias significativas entre las varianzas, es decir que son iguales. Una vez tenemos esta información pasamos directamente a la columna de varianzas iguales para la prueba $\mathrm{T}$ y comprobamos que el resultado de la prueba $t(0,720)$ da una probabilidad (sig. bilateral) de 0,475 , superior, por tanto, al 0,05. Además el intervalo confidencial (de -1,15200 a 2,42906) incluye el valor cero. Todo ello nos permite concluir que no hay diferencias significativas entre las medias alcanzadas por los grupos experimental y control en la nota total alcanzada en la primera prueba. 


\subsection{Resultados de la prueba específica de lectura}

Como ya se detalló anteriormente, la prueba específica de textos de Oceanografía Física estuvo dividida en dos partes. La primera constaba de 7 ítems y la segunda de 10. Cada una de estas dos partes se correspondía con dos tareas diferentes. En la primera de ellas, el alumnado debía hacer un resumen en su lengua materna de cada uno de los siete extractos dados. Mientras que en la segunda, el alumnado debía ubicar diez términos en el hueco correcto de 10 pequeños textos. Consecuentemente, estamos ante una prueba que consta de 17 ítems.

Una vez que el test fue llevado a cabo, se pasó a su corrección, poniendo especial atención en la primera parte puesto que se prestaba a valoraciones más subjetivas. Para evitar ese posible error se optó por hacer una doble valoración por dos evaluadores independientes. En los casos en los que surgieron diferencias se calculó la media entre ambas puntuaciones. La segunda parte no presentó ninguna dificultad al existir tan solo una única posibilidad de ubicar cada término en los huecos asignados en el texto.

\subsubsection{Análisis descriptivo del grupo experimental y control}

Empezaremos presentando los datos para cada grupo en la primera de las tareas de lectura a través una tabla de contingencia (Tabla 4).

Si consideramos la nota media de esta prueba (3,50 puntos), se puede notar la superioridad en las calificaciones de los alumnos del grupo experimental: 15 alumnos superan dicha nota, por tan solo siete del grupo control. Pero si ponemos un nivel de comprensión más alto, puesto que se pretende que el alumnado estudie esta asignatura a través de los textos en lengua extranjera, y determinamos que se debe entender al menos cinco de los siete textos dados, comprobamos que ocho alumnos del grupo experimental lo consiguen, por solo dos del grupo control.

Tabla 4 de contingencia LECTESP1* GRUPO

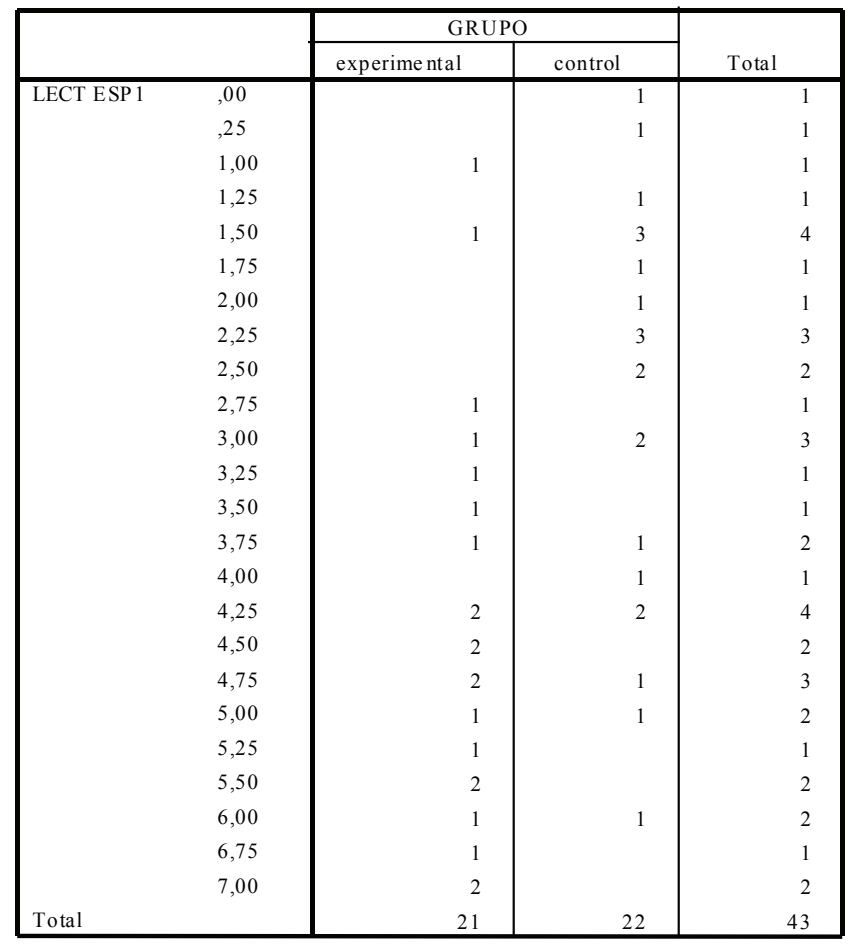


Para la segunda tarea de lectura los datos correspondientes a cada grupo son los que se especifican en la tabla de contingencia número 5.

Tabla de contingencia LECTE SP2 * GRUPO

\begin{tabular}{|cc|r|r|r|}
\hline & & \multicolumn{2}{|c|}{ GR UPO } & \multirow{2}{*}{ Total } \\
\cline { 3 - 4 } & & experimental & control & 3 \\
& 1 & 2 & 7 \\
& 1,00 & 1 & 6 & 5 \\
& 2,00 & 3 & 2 & 14 \\
& 3,00 & 7 & 7 & 5 \\
& 4,00 & 3 & 2 & 4 \\
& 5,00 & 1 & 3 & 2 \\
& 6,00 & 2 & & 1 \\
& 7,00 & 1 & & 2 \\
& 8,00 & 2 & & 43 \\
\hline
\end{tabular}

Se puede observar que de los diez ítems totales, la nota máxima alcanzada fue de ocho. Es evidente, según se ve en la tabla, que las mejores calificaciones fueron a parar a los alumnos del grupo experimental (seis ítems, dos estudiantes; siete ítems, uno; y ocho ítems adivinados, dos alumnos). En esta prueba, si estimamos la media en el 5 tenemos que seis alumnos del grupo experimental la rebasaron y sólo tres del grupo control, dándose además la circunstancia de que estos tres últimos alumnos obtuvieron solamente un cinco.

\subsubsection{Comparación estadística de las medias obtenidas}

Para la comparación de medias se utilizó la prueba T de muestras independientes que fue llevada a cabo con el programa SPSS.

En primer lugar se obtuvo la media, desviación típica y error típico de la media para cada uno de los dos grupos tal como se refleja en la Tabla 6.

Tabla 6. Estadísticos de grupo

\begin{tabular}{|ll|r|r|r|r|}
\hline & GRUPO & $\mathrm{N}$ & Media & $\begin{array}{c}\text { Desviación } \\
\text { típ. }\end{array}$ & $\begin{array}{c}\text { Error típ. de } \\
\text { la media }\end{array}$ \\
\hline TOTAL2 & experimental & 21 & 8,2262 & 3,19365 &, 69691 \\
& control & 22 & 5,1591 & 2,73268 &, 58261 \\
\hline
\end{tabular}

En la tabla anterior se puede apreciar que la media aritmética obtenida por el grupo experimental supera en más de tres puntos a la alcanzada por el grupo de control. Lo que nos falta por averiguar es si esta diferencia entre las dos medias es o no significativa. Para averiguarlo se utilizó, como ya hemos comentado, la prueba T de muestras independientes que nos aportó los resultados que presentamos en la tabla que presentamos a continuación. 
Tabla 7. Prueba T de muestras independientes para la comparación de resultados

\begin{tabular}{lll}
\hline & & Varianzas iguales \\
& & \\
\hline \multirow{2}{*}{$\begin{array}{l}\text { Prueba de Levene para la igualdad } \\
\text { de varianzas }\end{array}$} & F &, 344 \\
& Sig. &, 561 \\
\hline \multirow{2}{*}{ Prueba T para la igualdad de medias } & T & 3,389 \\
& Gl & 41 \\
& Sig. (bilateral) &, 002 \\
& Diferencias de medias & 3,0671 \\
& Error típ de la diferencia &, 90502 \\
& Intervalo de confianza inferior & 1,23937 \\
& Intervalo de confianza superior & 4,89483 \\
\hline
\end{tabular}

Una vez más el tratamiento informático de comparación de medias se inicia con una prueba previa al tratarse de dos grupos independientes. En este caso la prueba de Levene para la igualdad de varianzas nos indica que el resultado obtenido $(0,344)$ tiene una probabilidad $(0,561)$ superior a 0,05 , lo que viene a confirmarnos que no hay diferencias significativas entre las varianzas de ambos grupos, es decir que son estadísticamente similares. Una vez aclarado este dato, podemos pasar directamente a la columna de varianzas iguales para la prueba $\mathrm{T}$ y constatamos que el resultado de la prueba T $(3,389)$ da una probabilidad (sig. bilateral) de 0,002 , inferior, por tanto, a 0,05. Además el intervalo de confianza (de 1,23937 a 4,89483) no incluye el valor cero. Todo ello nos permite concluir que sí hay diferencias significativas entre las medias alcanzadas por el alumnado del grupo experimental y el grupo de control en la prueba de lectura específica, siendo esta diferencia significativa favorable al grupo experimental.

\subsubsection{Búsqueda de correlaciones}

En nuestra investigación, además del interés por comparar medias, también nos interesaba comprobar si existía algún tipo de correlación entre los resultados obtenidos en cada uno de los dos test de lectura llevados a cabo: el de lengua inglesa y el de lectura comprensiva de textos científicos. Para conseguir este fin hemos utilizado el coeficiente de correlación $r$ de Pearson puesto que es el más usado cuando se comparan dos variables cuantitativas de intervalo como las que aquí se están manejando. Al pedir al programa estadístico SPSS que nos indicara el índice de correlación entre el primer test de lectura (de índole general) y el segundo (específico de Oceanografía Física) nos dio una correlación de 0,489, con una probabilidad (sig. bilateral) de 0,001. Es decir, se constata una correlación muy alta, al nivel 0,01 , entre las calificaciones de cada alumno en ambas pruebas.

\section{Conclusiones e implicaciones}

Todos los datos aportados por las diferentes pruebas estadísticas nos indican que el alumnado se ve significativamente favorecido al leer textos específicos cuando se le da un glosario de términos técnicos, siendo además esa ayuda valiosa para todo el alumnado. Es decir, tanto el alumno que tiene una competencia lectora en lengua extranjera baja como el que la tiene 
elevada mejora en su rendimiento aunque, lógicamente, se siguen marcando las diferencias de nivel. Sin embargo, se sigue apreciando que hay un sector del alumnado que aún no consigue tener un nivel de comprensión adecuado de los textos específicos y dicha incapacidad tiene su origen en el bajo nivel lingüístico de tipo general. Con lo que podemos concluir que son necesarias dos premisas para conseguir el objetivo de poder estudiar la asignatura de Oceanografía Física: disponer de un nivel intermedio (A2 según el Marco Europeo de Referencia para las Lenguas) en la lengua en que esté escrito el documento y, además, disponer de un glosario de términos específicos de la asignatura.

\section{Notas}

1. Bauman y Culligan (1995) adaptaron la versión de West de 1953 y con el fin de determinar qué palabras incluir como formas relacionadas con la palabra núcleo, usaron el estándar establecido por Bauer y Nation (1995). Es un conjunto de dos mil palabras seleccionadas por su frecuencia de uso.

2. Coxhead (2000) desarrolló este nuevo listado léxico académico a partir de un corpus de tres millones y medio de palabras extraídas de textos académicos.

\section{Bibliografía}

Alderson, J.C. y A.H. Urquhart (eds.). 1984. Reading in a foreign language. New York: Longman.

Barnett, M. 1986. "Syntactic and lexical/semantical skill in foreign language reading: importance and interaction". Modern Language Journal. 70: 343- 349.

Barr, R. et al. 1996. Handbook of reading research. New York: Longman.

Bauer, L. y P. Nation. 1995. “Word families”. International Journal of Lexicography. 6 (4): 253-279.

Bauman, J. y B. Culligan. 1995. General Service List. http://jbauman/gsl.html.

Berman, R. 1984. Syntactic components of the foreign language reading process. En: Alderson y Urquhar (eds.), 139- 156.

Bernhardt, E.B. y M. Kamil. 1995. "Interpreting relationships between L1 and L2 reading: consolidating the linguistic threshold and the linguistic interdependence hypotheses". Applied Linguistics. 16: 15- 34.

Block, E. 1986. “The comprehension strategies of second language readers”. Tesol Quarterly. 20: 463- 494.

Block, E. 1992. "See how they read: comprehension monitoring of L1 and L2 readers". Tesol Quarterly. 26: 319- 343. 
Carrell, P.L. 1991. "Second language reading: reading ability or language proficiency?" Applied Linguistics. 12: 159- 179.

Clarke, M. 1980. "The short circuit hypothesis of ESL reading or when language competence interferes with reading performance". Modern Language Journal. 64: 203- 209.

Cooper, M. 1984. "Linguistic competence of practiced and unpractised non-native readers in English". En: Alderson y Urquhar (eds.), 122- 138.

Cornaire, C. 1999. Le point sur la lecture. París: CLE International.

Coxhead, A. 2000. “A new academic wordlist”. Tesol Quarterly. 34: 213, 238.

Crow, J. y R. Quigley. 1985. “A semantic field approach to passive vocabulary acquisition for reading comprehension”. Tesol Quarterly. 19: 497- 513.

Cummins, J. 1980. "The cross-lingual dimensions of language proficiency: implications for bilingual education and the optimal age issue". Tesol Quarterly. 14: 175- 187.

Daneman, M. 1996. Individual differences in reading skills. En: Barr et al. (eds.), 512- 538.

Hee Ko, M. 2005. “Glosses, comprehension, and strategy use”. Reading in a Foreign Language. 17: $125-143$.

$\mathrm{Hu}$ Hsueh-Chao, M. y P. Nation. 2000. "Unknown vocabularydensity and reading comprehension". Reading in a Foreign Language. 13: 403- 430.

Huntley, S. y M. Peñate. 2003. "Specific reading at an advanced level: linguistic or strategic competence?". Ibérica (Revista de la Asociación Europea de Lenguas para Fines específicos). 5: 87- 100.

Rayner, K. y A. Pollatsek. 1989. The Psychology of Reading. Englewood Cliffs, NJ: Prentice Hall.

Rodríguez, A. y M. Peñate. 2008. "La investigación de los procesos lectores: tendencias y enfoques en la lectura general y específica". Revista de Lenguas para Fines Específicos. 13- 14: 241- 284.

Segalowitz, D. 1991. "Does advanced skill in a second language reduce automaticity in the first language?” Language Learning. 41: 59- 83.

Stanovich, K. 1986. "Matthew effects in reading: some consequences of individual differences in the acquisition of literacy". Reading Research Quarterly. 21: 360- 407. 
Upton, T.A. 1997. "First and second language use in reading comprehension strategies of Japanese ESL students." Teaching English as a Second or Foreign Language. 3: 1- 24.

Ward, A. 2000. Preparation PET and Practice. Oxford: Oxford University Press.

Ward, J. 1999. "How large a vocabulary do EAP Engineering students need?" Reading in a Foreign Language. 12: 309- 323.

West, M. 1953. A General Service List of English Words. London: Longman.

Williams, R. y D. Dallas. 1984. Aspects of vocabulary in the readability of content area L2 educational textbooks: a case study. En: Alderson y Urquhar (eds.), 199- 212. 